\title{
Deutsche Fachsprachen: alte und neue Erfahrungen mit dem Begriff, Kultur' in den Geisteswissenschaften und im Bereich Deutsch als Fremdsprache
}

\begin{abstract}
Maria J. P. Monteiro*
Abstract: In the last few years, the areas of study of German as Foreign Language and German for Specific Purposes have evidently made an inflationary, and sometimes even inadequate use of concepts related to the adjectives 'cultural' and intercultural'. The concepts have often been used so as to serve only to instigate the increase, permanency and dissemination of stereotypes and pre-conceptions. In the Winter 2006/07 Semester, a course called "Fachsprachen und Kultur" was offered on the way the concepts of culture are approached in different fields of study. This paper presents the conclusions of some of the participants on the use of the concept in their primary or secondary areas. The results show that it is urgent and necessary to examine the concepts of culture used in our field of study so that stereotypes do not become the basis for a didactics of German as a Foreign Language.
\end{abstract}

Keywords: German as Foreign Language; culture; pre-conceptions; stereotypes.

Resumo: Nas áreas de estudos Alemão como Lingua Estrangeira e Alemão para Fins Especificos é evidente, nos últimos anos, o uso inflacionário e até, às vezes, impróprio, de conceitos relacionados aos adjetivos cultural e intercultural. Freqüentemente, os conceitos são usados de tal forma que apenas servem para fomentar o crescimento, a fixação e a difusão de estereótipos e preconceitos. No semestre de inverno 2006/07 foi oferecido um curso denominado "Fachsprachen und Kultur" (Magisterstudiengang Deutsch als Fremdsprache), no qual deveriam ser estudados os conceitos de cultura usados nas diferentes disciplinas. Aqui são apresentadas as conclusões de alguns participantes sobre o uso do conceito nas suas áreas principais ou secundárias. Os resultados mostram que se faz urgente e necessário ocupar-se com os conceitos de cultura que vigoram em nossa disciplina, para que estereótipos não se tornem a base de uma didática do Alemão como Língua Estrangeira.

Palavras-chave: Alemão como Língua Estrangeira; cultura; preconceitos; estereótipos.

Stichwörter: Deutsch als Fremdsprache; Kultur; Vorurteile; Stereotype.

\footnotetext{
* Professora Associada do Departamento de Letras Anglo-Germânicas da Universidade Federal do Rio de Janeiro. liamon@centroin.com.br
} 


\section{Einleitung}

Diese Arbeit ist aus dem Wunsch entstanden, einige Ergebnisse aus einem Seminar an der Technischen Universität Berlin zu präsentieren. Unter dem Titel „Fachsprachen und Kultur“ fand im Wintersemester 2006/07 am Institut für Sprache und Kommunikation der Technischen Universität Berlin (Magisterstudiengang Deutsch als Fremdsprache) ein Hauptseminar statt, in dem die Studenten (mit DaF als Haupt- oder Nebenfach) als Aufgabe hatten, einen Beitrag zur Klärung der kulturellen Komponenten in ihren anderen Haupt- und Nebenfächern zu leisten.

Besonders bei dieser Zielgruppe war es mir sehr wichtig, die Aufmerksamkeit auf die Verwendung des Kulturbegriffs zu lenken - es sind letztlich diese Studenten, die später in ihrem Berufsleben (sei es als Professoren, Forscher, DaF-Dozenten - auch DAAD-Lektoren - überall auf der Welt) einen gewissen Einfluss auf die diesbezüglichen Diskussionen in unserem Fach haben werden. Die konkreten Gründe dafür, sich mit diesem Thema zu beschäftigen und in diesem Fall ein Hauptseminar zu diesem Thema anzubieten, sind in den letzten Jahren immer zahlreicher geworden, besonders durch die ständige Beobachtung, wie in der DaF-Literatur mit den Begrifflichkeiten aus diesem Umfeld umgegangen wird. Vor allem war es mir wichtig in dem Hauptseminar hervorzuheben, dass der Begriff „Kultur“ keinesfalls eindeutig und statisch ist.

Bereits 1992 hat sich Hess ausführlich mit dem Problem auseinander gesetzt und m.E. einen entscheidenden Beitrag zu dieser Diskussion geleistet. U.a. übt er harte Kritik an dem latenten Sendungsbewusstsein der Ansätze, die die Aufgabe der Germanistik darin sehen, DaF-Lernern zum Erkennen eigener Bedürfnisse und zum Erlangen einer kulturellen Mündigkeit zu verhelfen (Hess 1992:18ff.). Seine Arbeit wurde jedoch in den folgenden Jahren kaum erwähnt. Noch im Jahr 1997 stellte ALTMAYER fest:

\footnotetext{
In nahezu allen Veröffentlichungen ist von 'Kultur', von 'kulturspezifischen' oder 'kulturbedingten' Verhaltens- oder Denkmustern, von 'interkulturellem', 'kulturkontrastivem' oder 'kulturvergleichendem' Vorgehen, von der Dialektik von 'eigener' und 'fremder Kultur', von 'Kulturschock' oder 'Kulturverstehen' die Rede. (ALTMAYer 1997:1)
}

Die Tendenz hat sich nicht geändert, und das wird auch von den studentischen Arbeiten belegt. Bevor ich mich aber den studentischen Beiträgen widmen kann, werde ich mich sehr kurz mit dem Kulturbegriff im Allgemeinen (Teil 2) und mit dem Kulturbegriff in geisteswissenschaftlichen Fächern (Teil 3) beschäftigen. Im 3. Teil versuche ich auch, einen kurzen 
Überblick der unterschiedlichen Verwendungen dieses Begriffs im Fach Deutsch als Fremdsprache vorzustellen, um anschließend über die Beiträge einiger Studenten aus dem Seminar zu berichten (Teil 4).

\section{Der Kulturbegriff}

In der Regel weisen die Versuche, den Kulturbegriff zu definieren, zunächst einmal auf die alltagssprachliche Verwendung hin, wo sich unterschiedliche Facetten des Kulturbegriffs finden (HANSEN 2003:11ff): a) Kultur als Teil der Hochkultur der Künste und des Kulturbetriebs; b) Kultur als besonderer Lebensstil, als Kultiviertheit des sozialen Umgangs; c) Kultur als distinkte Lebensformen in Gemeinschaften und Ländern; d) Kultur als domestiziertes Naturphänomen (Landwirtschaft, Kulturlandschaft). Lexikalisch stammt der Begriff aus dem Lateinischen (colo, colui, colere), mit zwei unterschiedlichen Bedeutungen: a) pflegen, bebauen, bestellen (Ackerbau) und b) anbeten, verehren (Religion). Die Definitionen des Begriffs spiegeln verschiedene Theorien der Bewertung und des Verständnisses menschlicher Tätigkeit wider und die Definitionsmöglichkeiten sind nahezu unendlich.

HiNNENKAMP hatte bereits 1994 einen Versuch unternommen, den Begriff Kultur in Zusammenhang mit Kommunikation zu bringen, um einen Definitionszwang zu vermeiden (S. 6). Dabei formuliert er „eine Art minimales Vorverständnis oder implizite Vorannahmen“, ohne disziplinäre oder methodologische Unterschiede zu beachten:

- Es gibt unterschiedliche, voneinander unterscheidbare Kulturen.

- Kultur und Kommunikation stehen in einem Zusammenhang.

- Kommunikationsteilnehmer sind immer auch Teilnehmer bzw. Teilhaber einer Kultur.

- Kulturelles spiegelt sich in der Kommunikation wider

- Kulturteilhabe heißt: in einer spezifischen Weise kommunizieren.

- Gemeinsame Kulturteilhabe erleichtert die Kommunikation, unterschiedliche Kulturteilhabe erschwert sie.

In der Diskussion um den Begriff stellt HinnenKamp (1994: 6ff) unterschiedliche Sichtweisen, Empfindungen und Bewertungen des Begriffs dar, wobei immer deutlicher wird, wie schwierig es ist, Merkmale, Grenzen, Empfindungen und Wahrnehmungen von Kulturen zu bestimmen. 
Ein großer Teil der gegenwärtigen kulturtheoretischen Ansätze geht davon aus, dass Kultur weder einer bestimmten Region noch Gruppe zugewiesen werden kann. Damit werden so genannte essentialistische Kulturvorstellungen (teils auch als Container-Kultur bezeichnet) verabschiedet, die Kultur als ein wie auch immer abgrenzbares System verstehen (vgl. WAGNER 2002: 11): „Kulturen sind Produkt von Beziehungen und Durchquerungen und entwickeln sich erst im Kontakt mit dem Fremden. Kultur bedeutet immer schon ,zwischen den Kulturen'“.

HinNENKAMP (1994: 6ff) stellt die Frage, ob Kultur „als eine Art ausgehandeltes Wissensmanagement" anzusehen wäre - trotz der unleugbaren Tatsache, dass in den Kultur-, Sozial- und Sprachwissenschaften „dezidierte Begriffe von Kultur" vorhanden sind (hermeneutische, strukturalistische, semiotische, kognitive, analytische, normative usw.). Falls ja, sollte bei jedem Herangehen an diese Fragestellung zunächst einmal sehr deutlich gemacht werden, welcher Ansatz vorgezogen wurde. Wenn nicht, wäre die einzige Alternative die von RIETHMÜLLER (2005) dargestellte Sichtweise, die zu den interessantesten Ansätzen über die Beziehung Kultur-Gesellschaft zählt:

Auf kulturellem Feld schließlich besteht das Neue im Zusammenhang mit besagter

Überflutung des Denkens in einem fortschreitenden Flottieren von massenkulturellen Zeichen, die nicht mehr vorwiegend Signifikanten und Signifikate in eine bestimmte kulturtypische Beziehung, eine „Ordnung der Dinge“ bringen. Viel eher können wir sie als lose Kumulierungen ewig aufeinander verweisender Signifikantenketten beschreiben, die damit angesichts immer unterschiedlicher werdender Verständnishorizonte der Einzelnen weitgehend oder vollständig ohne benennbares Signifikat enden. (RIETHMÜLLER 2005: 39)

\section{Das Fremde und die Fremden: Der Kulturbegriff in den Geisteswissenschaften}

HANSEN (2003: 361ff) beschäftigt sich mit der Frage, was unter dem Begriff Kultur in den Geisteswissenschaften verstanden werden soll ${ }^{1}$. Er versucht u.a. herauszufinden, ob der Kulturbegriff den Gesellschaftsbegriff ersetzt hat, in dem er eine Antwort auf die Frage „Ist der context of culture zum führenden Paradigma der Geisteswissenschaften geworden?“ in Bezug auf vier Gruppen von Disziplinen formuliert.

In der ersten Gruppe würden sich die Disziplinen befinden, die sich direkt mit Kultur als ihrem eigenen Gegenstand beschäftigen:

\footnotetext{
${ }^{1}$ Es ist nicht meine Absicht und es wäre auch nicht möglich, in diesem kurzen Aufsatz die gesamte Breite der Ansätze und Autoren darzustellen, die sich mit dem Thema beschäftigen. Deshalb muss ich mich auf eine kleine Auswahl beschränken.
}

Pandaemonium germanicum 11/2007, 111-124 - www.fflch.usp.br/dlm/alemao/pandaemoniumgermanicum 
Verhaltensforschung und Biologie, soweit sie den kulturellen Teil des Menschen erfassen, Kulturanthropologie, Kulturphilosophie.

Die zweite Gruppe umfasst Fächer, die spezielle Kulturen betrachten, und die „die kulturelle Verschiedenheit, wie sie sich etwa in den Landeskulturen äußert" (HANSEN 2003: 366), also in den Stammes-, Landesund Nationalkulturen, in den Vordergrund stellen. Dazu gehören u.a. die Ethnologie, die Volkskunde sowie die Landeskunden bzw. cultural studies, die nationalen Literaturwissenschaften (die einen Zusammenhang zwischen Nation und Literatur herstellen) und die Kulturenvergleichende Psychologie.

Die dritte Gruppe umfasst Disziplinen, die sich auf das Gesellschaftliche konzentrieren - in dieser Gruppe befinden sich z.B. die Soziologie, die Geschichte und die Politologie.

Die vierte Gruppe besteht aus Disziplinen, die sich etwas vom Kulturbegriff $\mathrm{zu}$ entfernen scheinen (wie die Rechts- und Wirtschaftswissenschaften, die Linguistik und die Psychologie).

Den Kulturbegriff im DaF-Bereich, dessen Einordnung innerhalb der Klassifizierung HANSENs einige Probleme mit sich bringt, werde ich im Folgenden näher betrachten. Wie bereits in der Einleitung erwähnt, wurde der Gebrauch dieses Begriffs innerhalb der Literatur zum Lehren und Lernen des Deutschen als Fremdsprache nicht gerade von Klarheit gekennzeichnet. Die Situation hat sich in den letzten 10 Jahren weiterhin verschärft. Es fällt schwer zu verstehen, was mit dem Begriff ,interkulturelle Kommunikation“ gemeint ist. Rost-Rotн (1996) hat in einem ausführlichen Aufsatz die Relevanzbereiche für den Fremdsprachenunterricht herausgestellt und Untersuchungen zu ethnographischen Besonderheiten deutschsprachiger Interaktionen im Kulturvergleich analysiert, wobei die Anzahl der angesprochenen Autoren nicht immer einer Vielfalt der Ansätze entspricht.

Tatsache ist, dass die Autoren, die sich dem DaF-Bereich widmen, sich vom Kulturbegriff aus Disziplinen inspirieren lassen, die aus praktisch allen vier von HANSEN (2003) genannten Gruppen stammen - hier seien nur als Beispiel die Kulturanthropologie, die Ethnologie, die Volkskunde sowie die Landeskunden (cultural studies), die nationalen Literaturwissenschaften und die Kulturenvergleichende Psychologie erwähnt.

Die interkulturellen Wege scheinen nach wie vor unergründlich zu sein. Nachdem Wierlacher 1985 Das Fremde und das Eigene als Prolegomena zu einer interkulturellen Germanistik angekündigt hat, fragt BIELEFELD 1991 unter dem Titel Das Eigene und das Fremde nach einem neuen Rassismus in der Alten Welt. 
Allein im Bereich der Beziehungen zwischen China bzw. Brasilien und Deutschland kann man schnell den Überblick verlieren (zu der Auseinandersetzung mit dem Begriff im chinesischen Kontext vgl. STEINMÜLLER 2000). Die Beiträge bieten unterschiedliche Konzepte und Wahrnehmungen an. Aber nach wie vor gilt - heute noch, im Jahre 2007 - die Aussage von HEss:

Der Begriff „Kultur ist als empirische Kategorie keineswegs eindeutig definiert. Die Formel „Kultur“ erscheint im alltäglichen Sprachgebrauch in vielfältiger Form und wird als selbstverständlich akzeptiert. Auf recht ungenaue Weise werden wahlweise folkloristische Erscheinungen, literarische oder musikalische Produkte, Begrüßungsformen, Speisegewohnheiten, Tischmanieren oder Alltagshygiene mit dem Etikett „Kultur“ belegt. Auch in der Fremdsprachendidaktik taucht der Begriff „Kultur“ auf, um eine Vielzahl von Phänomenen unterschiedlichster Art aus dem materiellen und gedanklichen Lebensumfeld von Sprechern einer jeweiligen Ziel- oder Muttersprache zu kennzeichnen. (HESS 1992: 39)

\section{Das Seminar und die dort entstandenen Arbeiten}

In dem Hauptseminar „Fachsprachen und Kultur“ haben wir uns in der ersten Phase mit dem Kulturbegriff beschäftigt. Danach sollten sich die Studenten (in Einzelarbeit) auf die Suche nach dem Begriff in ihren Fächern begeben. Die daraus entstandenen Ausarbeitungen wurden als Referat oder schriftliche Arbeit vorgelegt. Je nach den Gesichtspunkten und Kriterien, unter denen sie betrachtet werden, könnten alle studentischen Arbeiten, die im Hauptseminar entstanden sind, den vier Gruppen - oder mindestens dreien von denen - untergeordnet werden. Es sind auch Fälle von Arbeiten, die in mehr als eine Gruppe passen würden, weil unterschiedliche Kriterien angewandt werden könnten. Einige von ihnen werden im Folgenden beispielhaft nach der Unterteilung von HANSEN (2003) dargestellt. Um die Anonymität der Studenten zu gewährleisten, werden sie mit den Buchstaben A bis $K$ gekennzeichnet ${ }^{2}$. Die von den Studenten zitierten Werke werden in den Fußnoten aufgeführt.

\subsection{Gruppe 1}

Zu der Gruppe 1 gehörte zweifellos die Arbeit von Student A aus der Philosophie der Kultur. In der Arbeit wurde beschrieben, wie sich Kulturphilosophen (insbesondere Ernst CASSIERER) mit dem Eigentümlichen des Menschen beschäftigen - als System menschlicher Tätigkeit, interpretiert als die Sphäre des „Menschseins“ - wie Kultur: Sprache, Mythos, Religion,

\footnotetext{
2 Die Gruppe bestand aus jungen (deutschen und ausländischen) Frauen und Männern, und ich habe vorgezogen, sie untereinander nicht zu unterscheiden.

Pandaemonium germanicum 11/2007, 111-124 - www.fflch.usp.br/dlm/alemao/pandaemoniumgermanicum
} 
Kunst, Wissenschaft, Geschichte. Student B untersuchte bei dem Philosophen Marek Jan SIEMEK ${ }^{3}$ (Forschungsschwerpunkte Sozialphilosophie und politische Philosophie) die Entwicklung des Kulturbegriffs als Ausdruck der Befindlichkeiten der polnischen Gesellschaft, wobei der Philosoph eine zeitliche Linie der Verwendung des Begriffs zeichnet.

\subsection{Gruppe 2}

In den Disziplinen, die der Gruppe 2 zugeordnet werden können, hat sich Student $C$ für ein spezielles Gebiet entschieden, nämlich die literarische und wissenschaftliche Geokulturologie ${ }^{4}$ - es geht dabei um Sibirien, das nicht als „reiner Kulturraum wie die Metropolen Moskau und Petersburg“ betrachtet wird. Hier ist der Raum nicht nur geographische Kulisse, sondern Element der Handlung, er wird zum Handlungs-Raum; Objekt der Darstellung wird die geopolitische Dynamik der Beherrschung des Raumes und somit die geopolitische Dimension von Literatur als kulturelle Praxis.

Student $\mathrm{D}$ hat in dem Fach Psychologie einen Beitrag aus der kulturvergleichenden Psychologie ${ }^{5}$ ausgesucht, der in dem Bereich Deutsch als Fremdsprache und auch in der sog. Interkulturellen Kommunikation, wie sie sich in der Wirtschaft entwickelt hat, eine besondere Rolle spielt. Danach ist Kultur ,ein universelles, für eine Gesellschaft, Organisation und Gruppe aber sehr typisches Orientierungssystem. Dieses Orientierungssystem wird aus spezifischen Symbolen gebildet und in der jeweiligen Gesellschaft (...) tradiert. Es beeinflusst das Wahrnehmen, Denken, Werten und Handeln aller Mitglieder und definiert somit deren Zugehörigkeit zur Gesellschaft". In diesem Ansatz werden die Begriffe der Kulturstandards (,alle Arten des Wahrnehmens, Denkens, Wertens und Handelns, die von der Mehrzahl der Mitglieder einer bestimmten Kultur für sich persönlich und andere als normal, selbstverständlich, typisch und verbindlich angesehen werden“, THOMAS 2003: 380) und des kulturellen Handels in der Psychologie (die auf die wissenschaftliche Beschäftigung mit psychischen Vorgängen im Kontext interkultureller Begegnung zwischen Menschen verschiedener Kulturen Begegnung, Kommunikation, Kooperation usw.) vorgestellt.

\footnotetext{
${ }^{3}$ „Kultur und Zivilisation. Zwischen Tradition und Modernität“. In: LAwATZ, A. und H. OrLOwsKI (Hrg.). Deutsche und Polen. Geschichte - Kultur - Politik. München 2003.

${ }^{4}$ Frank, Susi K.,,Dostoevskij, Jadrincev und Cechov als 'Geokulturologen’ Sibiriens“. In: Gedächtnis und Phantasma. München, Otto Sagner 2001.

5 ThOMAS, Alexander. Kulturvergleichende Psychologie. Göttingen/Toronto/Seattle: Hogrefe 2003. 
Die anderen Arbeiten, die sich ausdrücklich mit DaF beschäftigen, und die dieser Gruppe zugeordnet werden könnten, werde ich am Schluss dieses Abschnitts näher betrachten.

\subsection{Gruppe 3}

In dieser Gruppe haben sich die Studenten $\mathrm{E}$ und $\mathrm{F}$ mit dem Kulturbegriff in der Soziologie beschäftigt, wobei sie hauptsächlich die Probleme der Soziologie mit der Definition von Kultur und mit der Determinierung der Aufgaben der Soziologie in Bezug auf das Thema unter die Lupe genommen haben. Dazu einige der wichtigsten Aussagen: Die Soziologie hat sich in den letzten Jahren immer schwer getan, das Kulturelle in der Gesellschaft zu verorten, die Soziologie hat die Tendenz, Kultur in Zusammenhang mit einer normativen sozialen Ordnung zu sehen, und das Kulturelle auf gemeinsame Werte, Symbole und Normen zu beschränken, die die Gesellschaft zusammenhalten. Andere Themen, wie die soziale Ungleichheit, bringen die Soziologie auf andere Ansätze, wie z. B. BOURDIEUs Werkzeugkasten von Symbolen, Geschichten, Ritualen und Weltanschauungen, aus denen sich der Einzelne bedient, um Handlungsstrategien zu bilden. Das Individuum ist ein aktiver Handelnder, er wählt kulturelle Angebote aus und setzt sie ein.

Student $G$ hat in Soziologie und Wissenschaftspolitik die Auffassung LEPENIES $^{6}$ dargestellt, der mit dem Begriff der Übersetzbarkeit der Kulturen arbeitet. Er sieht Kulturen als relativ geschlossene gesellschaftliche Ausrichtungen, die sich sowohl durch geistige Haltungen und Einstellungen auszeichnen, als auch durch Resultate. Dieses Kulturverständnis zeichnet sich auch durch eine nach innen gerichtete Identitätsbildung, die nach außen abgrenzend wirkt. Kultur sei „keine Instanz, der gesellschaftliche Ereignisse, Verhaltensweisen, Institutionen oder Prozesse kausal zugeordnet werden können“.

Aus der Politikwissenschaft diskutiert Student $\mathrm{H}$ den Begriff der „politischen Kultur“, der sich völlig von dem alltäglichen Kulturbegriff unterscheidet; die zentrale Frage der politischen Kultur konzentriere sich auf die Bedeutung von Werten, Einstellungen und Meinungen der Individuen für das politische System. Politische Kultur setzt sich nach ALMOND ${ }^{7}$ aus einer Mischung vielfältiger Vorstellungen zusammen, die sich in ihrer Stabilität unterscheiden: Werte fungieren als Maßstab des politischen Handelns, sind

\footnotetext{
${ }^{6}$ LEPENIES, Wolf. „Die Übersetzbarkeit der Kulturen. Ein europäisches Problem, eine Chance für Europa.“

7 Almond, Gabriel und Sidney Verba (ed.). The Civic Culture. Political Attitudes and Democracy in Five Nations. Paperback, 1989.
}

Pandaemonium germanicum 11/2007, 111-124 - www.fflch.usp.br/dlm/alemao/pandaemoniumgermanicum 
situationsübergreifend und objektunspezifisch, werden abstrakt formuliert, sind äußerst stabil; Einstellungen werden aus Werten abgeleitet, sind situations- und objektbezogen, tragen ein hohes Maß an Stabilität; Meinungen sind situationsabhängige Äußerungen, sind relativ leicht veränderbar. Das interdependente Zusammenspiel der drei Elemente formt die politische Kultur eines Systems.

\subsection{Gruppe 4}

Im Bereich der Wirtschaftswissenschaften oder, besser gesagt, in der Welt der Wirtschaft, sind viele interessante Erscheinungen um den Kulturbegriff entstanden. Student I hat den Begriff der Unternehmenskultur (oder auch Organisationskultur) näher untersucht ${ }^{8}$. Sie sei der im Unternehmen gewachsene Bestand von Basisannahmen (gemeinsame Vorstellungen über die Umwelt, die Erfassung der Realität, Verhaltensorientierungen usw.), Werten und Normen sowie Zeichen und Symbolen (als Ausdruck der und Identifikationsmuster für die Werte und Normen). Sie sei ein im Wesentlichen kollektives Phänomen, mit Ideen, Vorstellungen und Werte, die die Organisationsmitglieder gemeinsam verfolgen, ohne sich dessen unbedingt bewusst zu sein. Studien aus diesem Bereich $^{9}$ beschäftigen sich mit nationalen Unternehmenskulturen und vergleichen sie untereinander - z.B. zeigt eine Befragung, „dass die deutsche Unternehmenskultur die traditionellen Elemente der japanischen mit den modernen Merkmalen der amerikanischen Unternehmenskultur in gemäßigter Form verbindet“.

\subsection{Und wohin mit DaF?}

Die Arbeiten von Student J und Student K könnten unter Gruppe 2, als Teil der Volkskunde/Landeskunde/Germanistik oder unter Gruppe 4 als Teil der (Angewandten) Linguistik untergeordnet werden. Es ist aber schwierig, diesen Aspekt der Disziplin Deutsch als Fremdsprache irgendwo zu verorten.

Diese Schwierigkeit hängt meiner Meinung nach mit der Tatsache zusammen, dass die Autoren sich nicht mit didaktischen und methodischen Aspekten des DaF-Unterrichts beschäftigen, sondern mit Analysen aus ihren anderen früheren Studienbereichen - aus der Soziologie, aus der Kontrastiven Linguistik, aus der Kommunikationstheorie, Politologie usw. - und diese

\footnotetext{
${ }^{8}$ SCHREYÖGG, Georg. Organisation. 2. Auflage, 1998, S. 444.

9 Bertelsmann StiftUnG. „Global operierende Konzerne erkennen ökonomische Relevanz der Unternehmenskultur". 2.07.04. Abrufbar unter http://www.uni-protokolle.de/nachrichten/id/36216/ [19.06.2007]
}

Pandaemonium germanicum 11/2007, 111-124 - www.fflch.usp.br/dlm/alemao/pandaemoniumgermanicum 
werden ohne Weiteres in den Unterrichtsraum transportiert. Die Begriffe, die dann verwendet werden, könnten als ethnologisch, soziologisch, anthropologisch und vieles Andere klassifiziert werden - wobei zu beachten ist, dass es allen Klassifizierungen an wissenschaftlichen und theoretischen Begründungen fehlen würde, da sehr zweifelhafte Komponenten hineinfließen, und zwar aus dem Bereich der Interkulturellen Germanistik. HESs hat bereits darauf hingewiesen (1993: 109f.):

\begin{abstract}
Die Begriffe „Kultur“ und „Tradition“ sind in der DaF-Didaktik durch den Einfluß der „Interkulturellen Germanistik“ in den letzten zehn Jahren weit verbreitet worden. Kaum eine neuere Publikation kommt ohne dieses Etikett aus. Hinter der „Interkulturellen Germanistik“, aber nicht nur durch sie vertreten, steht ursprünglich ein nobles Anliegen emanzipatorischer DaF-Pädagogik, nämlich: die Lebenssituation, die Sicht- und Denkweisen von Studenten zu berücksichtigen, sie „als sie selbst“" zu Wort kommen zu lassen, anstatt sie „kulturell fremdzusteuern“. Die „Interkulturelle Germanistik“ hat aus diesen Grundgedanken jedoch m. E. ein waberndes Hirngespinst von dichotisch gedachten „Eigen- und Fremdkulturen“ gemacht, das in seiner didaktischen Konsequenz genau das vermissen lässt, worauf es ursprünglich ankam: die Anerkennung sog. „fremder“ Lebenswelten als Grundlage von Unterrichtsplanung.
\end{abstract}

Student J hat eine kontrastive Studie von chinesischen und deutschen Buchrezensionen ${ }^{10}$ untersucht und ist zu dem Schluss gekommen, dass die chinesischen Rezensionen im Vergleich zu den deutschen immer eine Tendenz zur positiven Bewertung beinhalten, die eben der chinesischen Eigenschaft entsprechen, immer höflich zu sein. Als Beispiel wird ein Zitat aufgeführt: „Freilich hat das Buch auch einige nicht zufriedenstellende Punkte. [...] Aber insgesamt gesehen waren das bloß einige kleinere Einzelfehler in einem ausgezeichneten Gesamtwerk. Das Buch [...] ist wirklich eine sehr gelungene Monographie, die den Lesern sehr zu empfehlen ist“. Als Gegensatz wird aus einer deutschen Rezension zitiert: „[...] sei wenigstens an dieser Stelle ausdrücklich gesagt, dass ich die Kapitel 1-3, 5, 6, 9 und 11-13 durchaus lesenswert finde. Dennoch kann ich das Buch dem an Spracherwerb interessierten Leser ohne Vorkenntnisse nicht generell als Einführung empfehlen, weil es zu viele Fehler und Halbwahrheiten enthält.“

Aus diesen und anderen Beispielen wird entnommen, dass die Chinesen (eindeutig als eine homogene Masse dargestellt) als sehr höflich bezeichnet werden - weil sie immer positive Aspekte vor den negativen hervorheben -, während die Deutschen (auch eine homogene Masse) sehr grob mit den Berufsgenossen umgehen. Die Deutschen seien u.a. auch ironisch (Beispiel:

10 LIANG, Yong. „Höfflichkeit als interkulturelles Verständigungsproblem. Eine kontrastive Analyse Deutsch/Chinesisch zum kommunikativen Verhalten im Alltag und Wissenschaftsbetrieb“. In: Jahrbuch Deutsch als Fremdsprache 18 (1992), S. 65-86. Diese Angabe gilt für alle Zitate aus dieser Arbeit. 
„Für mich als Rezensentin ist nach Lektüre des ersten Teils jedoch nicht klarer geworden, wer nun wirklich der Adressat dieses Buches sein soll.“).

Student K hat sich das Thema „Sprache, Literatur und der Gebrauch des Kulturbegriffs im Fach DaF - Brasilien/Deutschland“ ausgesucht. Dabei wird der Kulturbegriff im Fach Deutsch als Fremdsprache, der von Altmayer (1997) dargestellt wird, auf die sprachliche Verwendung sowohl in der mündlichen Kommunikation als auch in der Literatur angewendet. Ich berichte hier nur über die Ergebnisse aus dem ersten Bereich, ohne auf die Literatur einzugehen. Sie beziehen sich auf so genannte „Kommunikationsstile“ der Deutschen und der Brasilianer, wie sie von SCHRÖDER (2006) ${ }^{11}$ betrachtet werden. Die Autorin sammelt - z.T. auf der Basis von soziologischen Theorien aus den 70er Jahren - Beweise dafür, dass Brasilianer einen barocken, narrativen, konkreten und oral geprägten, während Deutsche einen eher distanzierten, synthetischen, abstrakten und literarisch geprägten Kommunikationsstil aufweisen.

Die Autorin zählt u. a. folgende Beobachtungen auf (S. 54f), die diese Schlussfolgerungen unterstützen: a) „Viele Entscheidungen, Meinungen und Wünsche für die Zukunft sind im brasilianischen Textkorpus existentiellidealistisch und gefühlsmäßig, im deutschen dagegen pragmatisch-rationell motiviert.“; b) „In den Antworten der brasilianischen Interviews dominiert die Ebene des subjektiven Erlebens mit einem narrativen Erzählstil gegenüber einem stärker objektivierten Abstraktionsniveau in den deutsche Stellungsnahmen (...); c) „In den brasilianischen Interviews dominiert der additive Erzählstil gegenüber einer deutschen Verfahrensweise, die mit Hilfe von kausalen, konzessiven und konditionalen Subjunktoren der Schriftsprache (...)“; d) „Im Gegensatz zur brasilianischen Attribuierung enthält die deutsche Sprache ein sich stetig ausweitendes Arsenal an Komposita“, und deswegen seien die Antworten der Deutschen „knapp“ und „ausgefeilter“, dagegen die der Brasilianer „ausschweifend“.

\section{Abschließende Bemerkungen}

In diesem Aufsatz habe ich versucht, einige studentische Arbeiten aus dem Hauptseminar „Fachsprachen und Kulturen“ und damit die problematische Beziehung zwischen Kultur und Sprache in den Geisteswissenschaften und insbesondere im DaF-Bereich zu präsentieren.

\footnotetext{
11 SCHRÖDER, Ulrike. „Deutsche und brasilianische Kommunikationsstile im interkulturellen Vergleich“. In: Zeitschrift für Angewandte Linguistik 44, vol. 2, Februar 2006, p.49-69.

Pandaemonium germanicum 11/2007, 111-124 - www.fflch.usp.br/dlm/alemao/pandaemoniumgermanicum
} 
Laut den Studenten war ein sehr wichtiges Ergebnis aus dem Hauptseminar, dass sie zum ersten Mal über die Problematik nachgedacht haben, da sie bis zum Zeitpunkt unserer Diskussionen ,Kultur' als eindeutigen Begriff betrachtet hatten. Die Annahme, dass sie den Kulturbegriff aus den anderen Studienfächern auf das Verständnis des Bereiches Deutsch als Fremdsprache ohne weitere Überlegungen übertragen konnten, war für sie selbstverständlich.

Die vielfältige Verwendung des Kulturbegriffs in Zusammenhängen wie interkulturelle Kommunikation, interkulturelles Training, interkulturelle Didaktik u.a. zeigt eine Unklarheit bei dieser Verwendung. Das ist jedoch ein grundsätzliches Problem, wie BUDIN zeigt:

\begin{abstract}
Kulturwissenschaftliche Forschungsansätze haben sich ebenso unterschiedlich entwickelt wie die ihnen eigenen Begriffe von Kultur. Dies gilt nicht nur im interlingualen Vergleich, was allein am terminologischen Unterschied zwischen ,cultural studies“ und ,Kulturwissenschaft ${ }^{\star}$ schon sehr deutlich wird, sondern auch innerhalb einer Sprache. Der theoretische, methodische, und somit auch sprachlich-terminologische Pluralismus ist bisher in den Kulturwissenschaften aber kaum reflektiert worden. (BUDIN 1999)
\end{abstract}

Und auch nicht im DaF-Bereich. Hier gilt noch einmal die Aussage BuDINs, die sich ausdrücklich auf die philologischen Fächer bezieht:

Ein weiteres Problem der Kulturwissenschaft ist in ihrer einzeldisziplinären Verfangenheit $\mathrm{zu}$ sehen. Die inflationäre Verwendung des Terminus ,Kulturwissenschaft' in letzter Zeit lässt sich auf die vielzitierte Krise der Sozialund Geisteswissenschaften zurückführen: philologische Fächer etwa sehen den Ausweg aus ihrer Krise darin, sich fortan als Kulturwissenschaft zu bezeichnen. Instituts- oder Fakultätsbezeichnungen werden entsprechend geändert. Doch Namenskosmetik führt nicht aus Krisen; dies kann man nur erreichen, wenn (z. T. längst überkommene) Strukturen geändert bzw. neue Strukturen geschaffen werden. (BUDIN 1999)

Im DaF-Bereich gibt es offensichtlich kein Interesse daran, zweifelhafte Auffassungen durch neue, brauchbare Konzepte zu ersetzen. Nationale Identitäten werden immer noch als Block betrachtet. Deutsche seien gnadenlos offen und direkt (und deswegen ehrlich und vertrauenswürdig), ironisch, kritisch, rationell, kohärent; Ausländer seien nicht dazu fähig, abstrakt zu denken (MONTEIRO 1984: 474), unkritisch, unehrlich, unzuverlässig. Zum Teil entstehen solche Behauptungen als Ergebnis eines „interkulturellen“ Vergleichs unter Anwendung unzulässiger, als ,empirisch' dargestellter Methoden, wie in SCHRÖDER 2006, wo die Brasilianer noch einmal als homogene Masse dargestellt werden. Wer Brasilien etwas näher kennt, ist sich der Größe des Landes und der Vielfalt der „Brasilianer“ bewusst. Es wird deshalb dem Leser schwer fallen, Aussagen über „die 
Brasilianer“ Glauben zu schenken, die aus Antworten von ,intrakulturellen Gruppen“ (zusammengesetzt aus 200 Studenten und 200 Nicht-Studenten von irgendwo aus Brasilien) entstanden sind (SCHRÖDER 2006: 51ff.) $)^{12}$.

Tatsache ist, dass es in der Literatur zum DaF-Unterricht seit Jahrzehnten Arbeiten gibt, wie die beispielhafte Arbeit von HESs (1992), die bis ins letzte Detail zeigen, wie schwach die Grundlagen derartiger Aussagen sind. Eine seiner Schlussfolgerungen:

Zur Beschreibung von Unterrichtsvoraussetzungen ist „Kultur“ ein unnötiger Begriff, da die jeweiligen Bedingungsfaktoren auch durch andere, z. B. soziologische und didaktikwissenschaftliche Termini ausreichend erfasst werden können. In der Ausprägung, die er in der DaF-Diskussion der letzten zehn Jahre erhalten hat, suggeriert der Begriff m.E. unzulässigerweise die Möglichkeit zu summarischer Vereinfachung der Komplexität des Wirkungsgefüges vor Ort (1992: 52).

Leider gibt es bisher nicht viele Arbeiten, die sich mit dem Thema beschäftigen und nicht in die Falle der interkulturellen Homogenität tappen. Die Arbeiten von HESs sind ein Beispiel für diese wenige Arbeiten. Aus Brasilien zitiere ich ein einziges Beispiel: In einem Aufsatz stellt MeIRELES (2001) die Ergebnisse einer Untersuchung über die Verwendung der Negation im Deutschen und im brasilianischen Portugiesischen. Die Autorin macht darauf aufmerksam (MeIRELES 2001: 161), wie die unterschiedliche Verwendung der Negation seitens der Brasilianer zur Aufrechterhaltung von Vorurteilen veranlassen könnte - wie zum Beispiel dass die Brasilianer nicht fähig dazu sind, nein zu sagen und deshalb auch nicht ehrlich seien ${ }^{13}$. Darauf zu beharren, immer wieder alte Vorurteile ins Leben zu rufen, bringt uns als Fach nicht nach vorne. Wir waren und sind schon viel weiter.

\section{Literaturverzeichnis}

Altmayer, Claus. „Zum Kulturbegriff des Faches Deutsch als Fremdsprache“. Zeitschrift für Interkulturellen Fremdsprachenunterricht [Online], 2(2), 1997, 25 pp. http://www.spz.tu-darmstadt.de/projekt ejournal/ig 02 2/beitrag/almayer3.htm [12.09.2006]

Bielefeld, Uli (Hg.). Das Eigene und das Fremde. Nener Rassismus in der Alten Welt? Hamburg, Junius, 1991.

12 Es wäre m. E. absolut notwendig, zumindest einige Informationen über die Städte/Regionen, Studienfächer u.a. zu liefern. Z.B. Studenten der brasilianischen Literatur aus einer kleinen privaten Universität im Hinterland haben bestimmt andere Kommunikationsstile als Betriebswirtschaftsstudenten oder Medizinstudenten in einer staatlichen Hochschule in der Hauptstadt São Paulo.

13 Im Original: "Parece-nos que a expressão sintática imprópria da Rejeição, Dissenção e Oposição em português e alemão é uma possível fonte de xenismos, gerando irritação nos falantes nativos e levando à formação de preconceitos quanto ao falante estrangeiro". (...) “Assim, do ponto de vista dos falantes alemães, os falantes brasileiros seriam 'incapazes de dizer não' e, por conseguinte, dissimulados”. 
BUDIN, Gerhard. „Sprache und Erkenntnis in den Kulturwissenschaften“. In: Internationale Kulturwissenschaften. http://www.inst.at/studies/s 0101 d.htm, 1999 [19.02.2006]

HAnSEN, Klaus P.. Kultur und Wissenschaft: Eine Einführung. 2. Aufl. Tübingen, Francke, 2003 (UTB 1846).

Hess, Hans-Werner. „Die Kunst des Drachentötens": zur Situation von Deutsch als Fremdsprache in der Volksrepublik China. München, iudicium, 1992.

Hess, Hans-Werner. „Kultur' und Zweitspracherwebsforschung“. In: STEINMÜLLER, Ulrich (Hrsg.). Deutsch international und interkulturell. Frankfurt, Verlag für Interkulturelle Kommunikation, 1993.

HinnENKAMP, Volker. Interkulturelle Kommunikation. Heidelberg, Julius Groos, 1994.

Meireles, Selma M. “A negação sintática em diálogos do alemão e do português do Brasil". In: Pandaemonium germanicum 5/2001, 139-168.

MONTEIRO, Maria. „Überlegungen und Vorschläge für eine Fremdgermanistik (oder für fremde Germanisten?)“. In: Info DaF 16, 4, 1989, S. 472-475.

RIETHMÜLLER, Jürgen. Kontrollgesellschaft außer Kontrolle - Perspektiven kritischer Theorie im Zeitalter der Globalisierung. Stuttgart, Merz Akademie, 2005.

Rost-Roth, Martina. (1996). „Deutsch als Fremdsprache und interkulturelle Kommunikation". In: Zeitschrift für Interkulturellen Fremdsprachenunterricht [Online], 1(1), $\quad 37 \quad$ p. $\quad$ Abrufbar unter: http://www.spz.tudarmstadt.de/projekt ejournal/ig 01 1/beitrag/rost11.htm [12.01.2007]

SCHRÖDER, Ulrike. „Deutsche und brasilianische Kommunikationsstile im interkulturellen Vergleich“. In: Zeitschrift für Angewandte Linguistik 44, vol. 2, Februar 2006, p.49-69.

STEINMÜLLER, Ulrich: „Interkulturelle Kommunikation und Wissenschaftskooperation zwischen Deutschland und China“. In: Wissenschaftlicher Verlag der TongjiUniversität Shanghai (Hg.). Arbeiten zur Interkulturellen Kommunikation ChinesischDeutsch. Shanghai: Tongji-Universität, 2000.

STEINMÜLLER, Ulrich (Hrsg.). Deutsch international und interkulturell. Frankfurt, Verlag für Interkulturelle Kommunikation, 1993.

WAGNER, Bernd. „Kulturelle Globalisierung“. Aus Politik und Zeitgeschichte Band 12, 2002, p. 10-19. Bundeszentrale für politische Bildung. Abrufbar unter http://www.bpb.de/publikationen/VWSULT,0,0,Kulturelle Globalisierung.html [20.2.2007].

Wierlacher, Alois (Hg.). Das Fremde und das Eigene. Prologomena zu einer interkulturellen Germanistik. München, iudicium, 1985. 